\title{
LITÍASE VESICULAR ASSINTOMÁTICA EM MULHERES: ASPECTOS EPIDEMIOLÓGICOS E CLÍNICOS
}

\author{
ASYMPTOMATIC GALLSTONES IN WOMEN: EPIDEMIOLOGIC AND CLINICAL \\ ASPECTS
}

\author{
Adilson Cunha Ferreira ${ }^{1}$; Francisco Mauad Filho ${ }^{2}$; Fernando Marum Mauad ${ }^{1}$; \\ Daniela de Abreu Barra ${ }^{4}$; Rogério Lino Mattos ${ }^{1}$; Isac Jorge Filho ${ }^{3}$
}

\begin{abstract}
RESUMO: Objetivo: Analisar variáveis clínicas e ultra-sonográficas como presença ou ausência de barro biliar, espessura da parede e medida transversal da vesícula biliar, idade, paridade, presença ou ausência de diabetes melitus associadas à litíase vesicular assintomática, bem como determinar a sua prevalência em pacientes submetidas ao exame ultra-sonográfico. Método: Foram analisadas, em estudo prospectivo, 265 pacientes do sexo feminino, atendidas na Escola de Ultra-sonografia e Reciclagem Médica de Ribeirão Preto - EURP, durante o período de janeiro a setembro de 2001. Resultados: Evidenciou-se diferença estatisticamente significativa relacionada à litíase da vesícula biliar e: espessura da parede da vesícula biliar, barro biliar, diâmetro transverso da vesícula biliar, faixa etária, paridade, passando de 4,1\% na nulíparas, para 39,1\% nas multíparas e diabéticas. A prevalência de litíase na vesícula biliar, em pacientes assintomáticas, foi de $14.7 \%$. Conclusão: A litíase vesicular assintomática em mulheres ocorre principalmente com o decorrer da idade e da paridade. Os achados ultra-sonográficos mais freqüentemente encontrados foram: presença de barro biliar e de espessamento da parede da vesícula biliar (Rev. Col. Bras. Cir. 2006; 33(4): 235-241).
\end{abstract}

Descritores: Litíase; Cálculos biliares; Vesícula biliar; Ultra-sonografia.

\section{INTRODUÇÃO}

Distúrbios do trato biliar afetam uma porção significativa da população mundial. Mais de $95 \%$ das doenças do trato biliar são atribuíveis à litíase da vesícula biliar ${ }^{1}$. De acordo com a evolução clínica, informações de exames de imagem ou anatomopatológicos, a litíase vesicular poderá se apresentar como: colecistite aguda, colecistite crônica ou assintomática, a qual se define pela presença de cálculos vesiculares não associados à sintomatologia ou à história de dor biliar, independente da presença ou não de sintomas inespecíficos como flatulência, intolerância a alimentos gordurosos e dispepsia ${ }^{2}$.

A prevalência de litíase vesicular é variável de acordo com o continente, país, estado e cidade. Pode variar, inclusive, de acordo com os grupos de pacientes analisados. França $^{3}$ analisando laudos de necropsias, encontraram prevalência de 19,5\% em cirróticos. Ferreira ${ }^{4}$, avaliando a contribuição da ultra-sonografia abdominal no seguimento de pacientes pós tratamento do câncer mamário, encontraram prevalência de $10 \%$ e Badalamenti ${ }^{5}$ encontrou uma prevalência de $28 \%$ em pacientes em diálise. Particularmente, a prevalência de litíase vesicular assintomática tem sido motivo de vários estudos em diversos países ${ }^{6,7}$. Acomete aproximadamente $10 \%$ das populações americana e inglesa ${ }^{7}$.

Em um trabalho prospectivo, Akute $^{8}$ et al. demonstraram que a prevalência global de litíase em mulheres grávidas, encaminhadas a um centro de ultra-sonografia, foi de $2.1 \%$. Chamam a atenção para a baixa prevalência nesse grupo, quando comparado aos 10 a 20\% na Europa e América do Norte. Afirmam, ainda, que a maioria dos casos, $95 \%$, ocorreu em mulheres assintomáticas, e alertam para a necessidade de outros estudos serem realizados na população geral.

Em contraste, na África, sua prevalência é baixa, embora isso varie de uma parte do continente a outra. A tribo Masai, leste da África tem prevalência próxima a zero².

No Brasil, esses estudos são escassos e limitados. Coelho ${ }^{9}$ et al., avaliando 1.303 vesículas biliares em autópsias, encontraram 7,8\% de cálculo biliar. Relatam que a incidência de cálculo biliar foi maior no sexo feminino, 5,3 $\%$, do que no masculino, 3,9\%. Esses mesmos autores, empregando a ultra-sonografia em indivíduos hospitalizados com mais de 20 anos de idade, determinaram que a prevalência de colelitíase, é de $14,8 \%{ }^{10}$ e na população geral, avaliando também por meio da ultra-sonografia, encontraram uma prevalência de $9,3 \%{ }^{11}$.

1. Docente da Escola de Ultra-sonografia e Reciclagem Médica de Ribeirão Preto (EURP).

2 Docente da Faculdade de Medicina da Universidade de Ribeirão Preto - UNAERP.

3. Professor Doutor em Cirurgia; Presidente da Sociedade Paulista de Gastroenterologia; Professor Responsável pela Disciplina de Cirurgia Geral da Universidade de Ribeirão Preto (UNAERP).

4. Pós- Graduanda do Departamento de Ginecologia e Obstetrícia da Faculdade de Medicina de Ribeirão Preto, Universidade de São Paulo.

Recebido em 03-03-06

Aceito para publicação em 07-04-06

Conflito de interesses: nenhum

Fonte de financiamento: nenhuma

Trabalho desenvolvido na Escola de Ultra-sonografia e Reciclagem Médica de Ribeirão Preto (EURP) e apresentado para Exame Geral de Qualificação em Nível de Doutorado, ao Departamento de Ginecologia e Obstetrícia da Faculdade de Medicina de Ribeirão Preto, Universidade de São Paulo (USP). 
Apesar de um significante número de pacientes portadores de litíase vesicular serem assintomáticos, ela pode acarretar algumas complicações ${ }^{6,12}$, sendo as mais freqüentes a: colecistite aguda, colecistite crônica, calcificação da vesicular biliar, carcinoma da vesícula biliar, icterícia obstrutiva, abscesso, perfuração, íleo biliar, pancreatite aguda e Síndrome de Mirizzi, dentre outras ${ }^{13-16}$. Muito embora importantes descobertas na área dos exames diagnósticos ocorridas no século XX, principalmente nas duas últimas décadas, como a aplicação da ultra-sonografia, tomografia computadorizada, coledocoscopia e técnicas radiológicas intervencionistas ${ }^{17}$, tenham levado a uma melhor compreensão dessas complicações e diagnósticos cada vez mais precoces, a evolução clínica dos pacientes com litíase vesicular assintomático permanece controversa ${ }^{6,18,19}$.

Diante do exposto, o objetivo desta pesquisa foi o de determinar, nas pacientes submetidas ao exame ultrasonográfico abdominal, ao vaginal e ao obstétrico, sem queixas ou antecedentes sugestivos de colecistite calculosa, a presença de litíase (cálculos) na vesícula biliar em um serviço de ultra-sonografia de Ribeirão Preto, São Paulo, Brasil. Analisar, nesse grupo de pacientes, variáveis ultra-sonográficas e clínicas que estivessem relacionadas à litíase: presença ou ausência de barro biliar, espessura da parede e diâmetro transverso da vesícula biliar, idade, altura, peso, raça, paridade e a presença ou ausência de diabetes melitus.

\section{MÉTODO}

Foram analisadas, prospectivamente, 265 pacientes do sexo feminino, atendidas na Escola de Ultra-sonografia e Reciclagem Médica de Ribeirão Preto (EURP). Os critérios de inclusão atingiram todas as pacientes que assinaram o consentimento informado, e que foram submetidas a exames ultrasonográficos por qualquer indicação, mas nunca por suspeita de doença biliar e que estivessem em jejum de, pelo menos, oito horas. Essas pacientes tiveram seus exames complementados pelo estudo ultra-sonográfico da região cor-

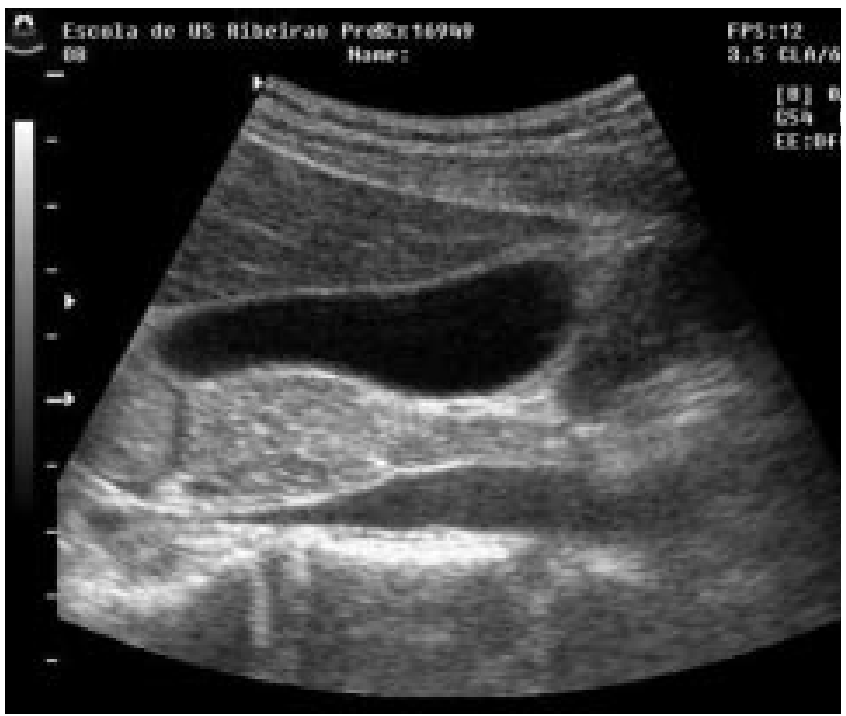

Figura 1 - Vesícula biliar normal avaliada ultra-sonograficamente com transdutor de $5 \mathrm{Mhz}$. respondente à vesícula biliar (Figura1). Os critérios de exclusão foram: preexistência comprovada de colelitíase, queixas sugestivas de colelitíase (especialmente dor no hipocôndrio direito e epigástrio), colecistectomia prévia, doença hemolítica, nefropatia instalada e dificuldade técnica.

O material da pesquisa foi constituído pelos relatórios dos exames ultra-sonográficos e dados clínicos da ficha da paciente. Os exames foram feitos por via abdominal, com aparelhos de ultra-sonografia com transdutor convexo, multifrequencial e banda larga de 3 a $6 \mathrm{MHz}$. Marca registrada Medison, modelo $9900^{\circledR}$, Hewlett Packard, modelo Image Point $^{\circledR}$, General Eletric, modelo Logic $400^{\circledR}$.

Os critérios para diagnóstico ecográfico foram:

Litíase da vesícula biliar - todas as imagens ecogênicas, produtoras de sombra acústica posterior e habitualmente móveis com as variações de decúbito (Figura 2).

- Barro biliar, também conhecido como lama biliar ou bile espessa, foi identificado por meio de ecos de baixa amplitude, formando, freqüentemente, uma imagem homogênea que se deposita na parede posterior, com nível líquido-líquido, deslocando-se lentamente, nas mudanças de decúbito, ou estando em suspensão, preenchendo, parcial ou totalmente, a luz da vesícula biliar, sem ocasionar sombra acústica (Figura3).

- Medida transversal da vesícula biliar - obtenção do maior diâmetro transversal da vesícula biliar, freqüentemente adjacente ao fígado, sendo mensurada somente a parte interna, sem envolver a parede (Figura 4).

- Espessamento da parede da vesícula biliar - esse critério foi avaliado em corte transversal, adjacente ao fígado, sendo mensurada a parede anterior e considerada espessa quando acima de $3 \mathrm{~mm}$. Foram validadas apenas medidas cujas vesículas, em seu diâmetro transverso interno, não eram inferiores a $2 \mathrm{~cm}$. Ou seja, vesículas adequadamente repletas para se afastarem os falsos positivos (Figura 4).

A análise estatística foi realizada por meio do teste do qui quadrado, para verificar a associação entre as variáveis; teste exato de Fisher, para avaliar se há ou não diferença significante, conforme variáveis, usando um $\mathrm{p}<0.001$; análise

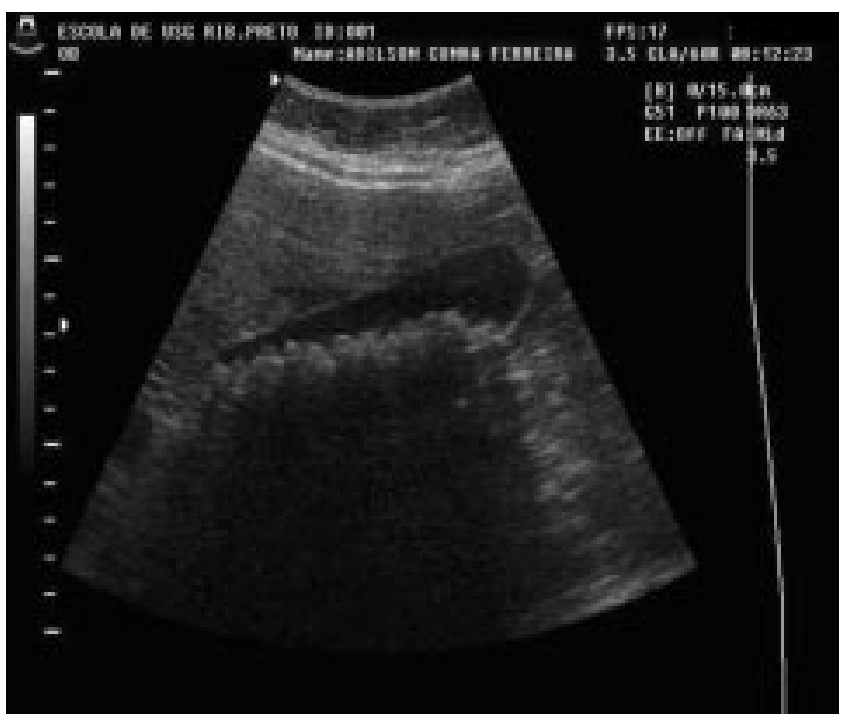

Figura 2 - Vesícula biliar preenchida por múltiplos pequenos cálculos avaliada ultra-sonograficamente com transdutor de $5 \mathrm{Mhz}$. 


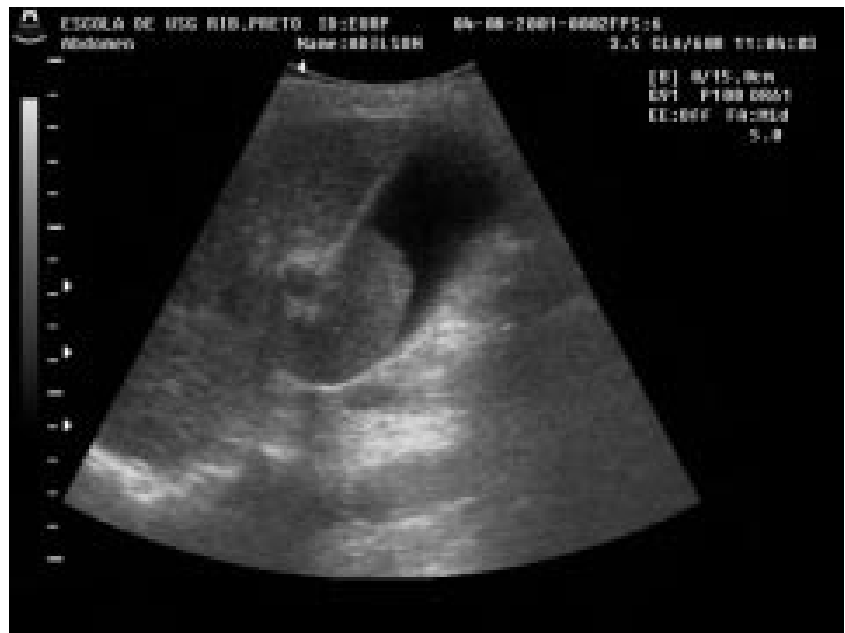

Figura 3 - Vesícula biliar preenchida parcialmente por barro avaliada ultra-sonograficamente com transdutor de $5 \mathrm{Mhz}$.

univariada, para se verificar a relação uma a uma com a variável litíase; análise de regressão logística multivariada, objetivando-se verificar como é a relação das variáveis com a variável litíase, quando são levadas em consideração todas as variáveis simultaneamente.

Este trabalho foi analisado pelo Comitê de Ética em Pesquisa, em sua $86^{a}$ Reunião Ordinária e enquadrado na categoria APROVADO, de acordo com processo HCRP n ${ }^{\circ}$ 5316/ 2001.

\section{RESULTADOS}

Do total de 265 pacientes que foram submetidas à análise ultra-sonográfica, 39 (14.7\%) apresentaram imagem ultra-sonográfica compatível com litíase vesicular.

Quando se analisou a relação entre litíase da vesícula biliar e parede da vesícula biliar espessada $(>3 \mathrm{~mm})$, por meio do teste do qui quadrado, encontrou-se uma diferença significativa $(\mathrm{p}<0.001)$ Tabela 1.

$\mathrm{Na}$ análise da relação entre litíase da vesícula biliar e barro biliar, há diferença significativa $(\mathrm{p}<0.001)$ tabela 2 .

A análise da variável medida transversal da vesícula biliar para quem tem cálculo versus quem não tem cálculo, por meio do teste T de Student, evidenciou uma média de $2,17 \mathrm{~cm}$, com desvio padrão de 0,56 , para quem não tem cálculo na vesícula biliar, quando comparado a uma média de $2,50 \mathrm{~cm}$, com desvio padrão de 0,73 , para quem tem cálculo na vesícula biliar. As pacientes com cálculo possuem em média valores

Tabela 1 - Relação entre parede espessada e presença ou não de litíase na vesícula biliar.

\begin{tabular}{lccrc}
\hline & \multicolumn{4}{c}{ Parede Espessada } \\
\cline { 2 - 5 } Litíase & $\mathbf{n}$ & \%im & n & \% \\
\hline Não & 5 & 35.7 & 221 & 88 \\
Sim & 9 & 64.3 & 30 & 12 \\
\hline
\end{tabular}

Teste qui quadrado $p<0.001$

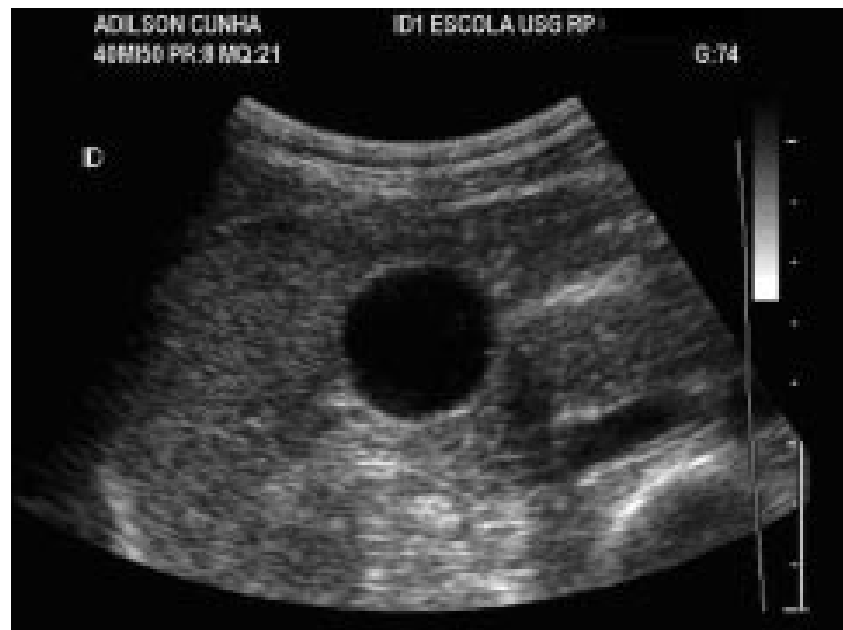

Figura 4 - Vesícula biliar local da medida transversal e análise da espessura da parede avaliada ultra-sonograficamente com transdutor de $5 \mathrm{Mhz}$.

superiores de medida transversal aos das pacientes que não possuem cálculo. $(\mathrm{p}=0.03)$.

Analisando-se a relação entre faixa etária e litíase da vesícula biliar, mostrou que os percentuais de presença de litíase variam significativamente, conforme muda a faixa de idade. Podese distinguir três situações: uma primeira faixa de prevalência zero entre os jovens abaixo de 20 anos, uma segunda faixa, entre 20 e 50 anos, cuja prevalência média gira em torno de $10 \%$, e a terceira, acima de 50 anos, com taxa acima de $42 \%$. Há diferença significativa $(\mathrm{p}<0.001)$ para as diversas faixas etárias, descrita anteriormente, conforme ilustra a Tabela 3.

$\mathrm{Na}$ análise da relação entre litíase da vesícula biliar e paridade, os percentuais de presença de litíase variam significativamente $(\mathrm{p}<0.001)$, conforme muda a faixa de paridade. Observa-se que, conforme aumenta a paridade, aumenta o percentual de ocorrência de litíase conforme ilustra a Tabela 4.

Quando se analisou a prevalência de diabetes na amostra encontraram-se $24(9,1 \%)$ pacientes, dentre as quais $13(54,2 \%)$ foram diagnosticadas litíase vesicular. A análise da relação entre litíase da vesícula biliar e diabetes, por meio do teste do qui quadrado, revelou diferença estatisticamente significativa $(\mathrm{p}<0.001)$ Tabela 5.

Do total da amostra, 45 pacientes encontravam-se grávidas sendo que, em duas $(4,4 \%)$ diagnosticou-se litíase.

A análise da relação entre litíase da vesícula biliar e raça, altura e peso da paciente não revelou diferença significativa. Os percentuais de presença de litíase são equivalentes, independentemente da raça, peso e altura.

Tabela 2 - Relação entre barro biliar e presença ou não de litíase na vesícula biliar.

\begin{tabular}{lrcrc}
\hline & \multicolumn{4}{c}{ Barro biliar } \\
\cline { 2 - 5 } Litíase & $\mathbf{n}$ & $\boldsymbol{\%}$ & $\mathbf{n}$ & $\mathbf{\%}$ \\
\hline Não & 8 & $38.1 \%$ & 218 & $89.3 \%$ \\
Sim & 13 & $61.9 \%$ & 26 & $10.7 \%$ \\
\hline
\end{tabular}

Teste qui quadrado $p<0.001$. 
Tabela 3 - Relação entre faixa etária e presença ou não de litíase na vesícula biliar.

\begin{tabular}{|c|c|c|c|c|c|c|}
\hline & \multicolumn{5}{|c|}{ Idade } \\
\hline & & $<20$ & {$[20-30$} & {$[30-40$} & {$[40-50$} & $>50$ \\
\hline \multirow[t]{2}{*}{ Litíase } & Não & $31(100 \%)$ & $71(87.7 \%)$ & $60(89.6 \%)$ & $41(89.1 \%)$ & $23(57.5 \%)$ \\
\hline & Sim & $0 \quad(0 \%)$ & $10(12.3 \%)$ & $7(10.4 \%)$ & $5(10.9 \%)$ & $17(42.5 \%)$ \\
\hline
\end{tabular}

Teste qui quadrado $p<0.001$.

Tabela 4 - Relação entre paridade e presença ou não de litíase na vesícula biliar.

\begin{tabular}{|c|c|c|c|c|c|}
\hline & & \multicolumn{4}{|c|}{ Paridade } \\
\hline & & $\mathbf{0}$ & $1-2$ & $3-4$ & $>4$ \\
\hline Litíase & $\begin{array}{l}\text { Não } \\
\text { Sim }\end{array}$ & $\begin{array}{r}71(95.9 \%) \\
3(4.1 \%)\end{array}$ & $\begin{array}{r}92(91.1 \%) \\
9(8.9 \%)\end{array}$ & $\begin{array}{l}46(74.2 \%) \\
16(25.8 \%)\end{array}$ & $\begin{array}{l}17(60.7 \%) \\
11(39.3 \%)\end{array}$ \\
\hline
\end{tabular}

Teste qui quadrado $p<0.001$.

Para realização da análise de regressão logística multivariada, foram selecionadas, para integrar o modelo inicial, todas as variáveis que, na análise univariada, tiveram relação significativa com a variável litíase, a saber:

Litíase $=$ constante + idade + parede da vesícula biliar espessada + diabetes + gravidez + paridade + barro biliar + medida transversal da vesícula biliar. Foram excluídas, sucessivamente, as variáveis que pouco contribuíram no modelo até restarem apenas as significativas. Portanto, essas variáveis são as que mais influenciam ou não na ocorrência de litíase na vesícula Tabelas 6 e 7).

Para um melhor entendimento, dividiram-se os resultados em três grupos. O Grupo I envolveu todas as pacientes; o Grupo II foi formado por pacientes com menos de 50 anos, e o Grupo III, por pacientes com 50 anos ou mais, conforme se pode observar nas Tabelas 8,9 e 10 .

\section{DISCUSSÃO}

A conduta diante do achado ocasional de cálculos na vesícula biliar, ou seja, naquelas pacientes assintomáticas, vem sendo discutida há muito tempo ${ }^{6,19}$. A possibilidade de complicações futuras tem sido avocada por muitos grupos, para indicar a colecistectomia profilática. Outros grupos opinam pela conduta expectante alegando que essa indicação é relativa e afirmando que o risco de complicações é menor que o risco da operaçãa ${ }^{16,20,21}$.

Tabela 5 - Relação entre diabetes e presença ou não de litíase na vesícula biliar.

\begin{tabular}{lrlrr}
\hline & \multicolumn{3}{c}{ Diabetes } & \multicolumn{2}{c}{ Não } \\
\cline { 2 - 5 } Litíase & $\mathbf{n}$ & \% & n & \% \\
\hline Não & 11 & $45,8 \%$ & 215 & $89.2 \%$ \\
Sim & 13 & $54,2 \%$ & 26 & $10.8 \%$ \\
\hline
\end{tabular}

Teste qui quadrado $p<0.001$.
Com a introdução da ultra-sonografia no diagnóstico da litíase da vesícula biliar, obtem-se, a cada dia, evidências de que a maioria dos cálculos da vesícula biliar são assintomáticos ${ }^{8,10,18}$. Em outros países, vários estudos procuram estudar a prevalência de cálculos biliares assintomáticos. No Brasil, esses estudos são escassos e limitados ${ }^{18}$.

Nesta análise, do total de 265 pacientes que foram submetidas ao exame ultra-sonográfico, 39 (14.7\%) apresentaram imagem de litíase vesicular. Esse resultado é semelhante ao encontrado por Coelho ${ }^{10}$. Cabe ressaltar, entretanto, que esses autores examinaram um grupo de pacientes hospitalizados, sendo sabidamente maior a incidência de litíase vesicular. Esses mesmo autores em publicação recente ${ }^{10}$, demonstraram que a prevalência na população geral gira em torno de $9,3 \%$. Quando comparados com literatura internacional, os resultados desta pesquisa estão compatíveis com a média encontrada, que é 10 a $20 \%$ na Europa e América do Norte ${ }^{8,16}$.

Quando se avaliou a variável espessamento da parede da vesícula biliar e a presença de litíase, encontrou-se um total de $14(5.3 \%)$ pacientes que apresentaram parede da vesícula espessada. Em $64.3 \%$, foram diagnosticados cálculos. Muito embora o espessamento da parede da vesícula biliar seja considerado um sinal inespecífico, podendo ser encontrado em um grande número de doenças, na grande maioria das vezes está relacionado à presença de litíase ${ }^{18}$. A relação inversa não é verdadeira para o grupo de pacientes assintomáticas. Das trinta e nove pacientes com diagnóstico ecográfico de litíase, apenas $23 \%$ apresentavam espessamento da parede da vesícula biliar. Tal resultado leva ao entendimento de que a maioria das pacientes portadoras de litíase assintomática não apresenta, ecograficamente, espessamento da parede da vesícula biliar. Embora o fato de se encontrar espessamento da parede da vesícula biliar não autorize, clinicamente, a se falar em colecistite crônica calculosa, visto que o termo colecistite, deve ser empregado para identificar os processos inflamatórios deste órgão, ecograficamente este sinal está relacionado ao processo inflamatório local, mesmo nas pacientes assintomáticas. Surge, portanto, diante nossos 
Tabela 6 - Odds ratio das variáveis parede espessada, barro biliar, idade e paridade em relação à litíase na vesícula biliar para todas as pacientes.

\begin{tabular}{lccc}
\hline Parâmetro & Odds Ratio & $\begin{array}{c}\text { I.C. 95.0 \% } \\
\text { Superior }\end{array}$ & Inferior \\
\hline Parede espessada & 15.0807 & 55.7637 & 4.0784 \\
Barro biliar & 11.4292 & 37.8847 & 3.4480 \\
Idade & 1.0444 & 1.0808 & 1.0093 \\
Paridade & 2.1437 & 3.4980 & 1.3137 \\
\hline
\end{tabular}

Tabela 7 - Odds ratio das variáveis parede espessada, barro biliar e paridade em relação à litíase na vesícula biliar para as pacientes com idade inferior a 50 anos.

\begin{tabular}{lccc}
\hline & \multicolumn{3}{c}{ I.C.95.0 \% } \\
Parâmetro & Odds Ratio & Superior & Inferior \\
\hline Parede espessada & 12.8643 & 51.7628 & 3.1971 \\
Barro biliar & 8.1852 & 36.2035 & 1.8506 \\
Paridade & 2.3842 & 4.0769 & 1.3943 \\
\hline
\end{tabular}

resultados, um questionamento. Mesmos nas pacientes assintomáticas o fato de o exame ultra-sonográfico revelar parede espessada, autoriza-nos a chamar esse quadro de "colecistite" crônica calculosa ou simplesmente litíase vesicular. Esse aspecto é de muita relevância, já que a entidade nosológica "litíase assintomática" é definida pela ausência de sintomatologia. Torna-se necessário enfatizarmos o emprego correto da nomenclatura, pois colelitíase ou litíase vesicular assintomática e colecistites agudas ou crônicas constituem condições que podem ter cursos diferentes ${ }^{13}$. Os pacientes que não têm sintomas, porém apresentam sinais ecográficos de parede da vesícula biliar espessada deveriam ser incluídos em um grupo separado de risco cirúrgico? Essa controvérsia ainda vai persistir. Mas, frente aos nossos resultados, a presença de parede da vesícula biliar espessada deve orientar o ultra-sonografista a rastrear cuidadosamente a vesícula biliar, visando à identificação de litíase e barro biliar.

Encontrou-se uma relação estatisticamente significativa para a relação barro e litíase da vesícula biliar. Dos 8,2 \% dos pacientes em que foi diagnosticado barro biliar ocorreu uma associação com litíase da vesícula biliar em 61,9\%. Tal resultado corrobora ainda mais, a etiopatogenia da morbidade em epígrafe, uma vez que os cálculos são constituídos, na grande maioria dos casos, por colesterol puro ou associado a pequenas quantidades de sais de cálcio e bilirrubina, além de traços de ácidos graxos, fosfolipídios, ácidos biliares e glicoproteínas ${ }^{18}$. No presente trabalho, 38,1\% dos pacientes tiveram diagnóstico ecográfico de barro biliar sem cálculos. As mulheres nas quais se diagnosticou barro biliar sem a presença de cálculos merecem acompanhamento sistemático, já que possuem maior possibilidade de formarem litíase da vesícula biliar com o passar do tempo e merecem tratamento, pois a "síndrome da bile espessa" pode causar manifestações clínicas relevantes ${ }^{18}$.

Embora a litíase da vesícula biliar possa ser observada em recém-nascido, na infância e adolescência, ela predomina a partir da quinta década de vida, aumentado progressivamente com a idade ${ }^{10}$. A prevalência aos 70 anos pode chegar a $40 \%{ }^{22}$. Analisando-se a relação entre faixa etária e litíase da vesícula biliar, observou-se que os percentuais de presença de cálculo variam significativamente conforme muda a faixa de idade. Foi possível distinguir três situações: uma primeira faixa de prevalência zero entre os jovens abaixo de 20 anos, uma segunda faixa, entre 20 e 50 anos, cuja prevalência média gira em torno de $10 \%$, e a terceira, acima de 50 anos, com taxa de $42,5 \%$. Os dados obtidos confirmam os achados de Coelho ${ }^{11}$ em que a idade média do grupo com litíase biliar foi de 59,9 anos.

Nesta análise, os percentuais de presença de litíase da vesícula biliar variam significativamente, conforme muda a paridade. Encontramos uma incidência em nulíparas de 4.1\%; em mulheres com até dois filhos, de $8.9 \%$; com três ou quatro filhos, de 25.8\%; e naquelas com mais de quatro filhos é de $39.3 \%$, chegando ao percentual de $71,4 \%$ se esse fato estiver associado ao diabetes. Esse resultado é de muita relevância, visto que as pacientes diabéticas com litíase vesicular apresentam uma mortalidade bem maior que as não diabéticas. Os diabéticos que desenvolvem colecistite aguda apresentam mortalidade de $10-15 \%{ }^{18}$.

Os resultados encontrados na literatura são uniformes quando abordam litíase da vesícula biliar e paridade, demonstrando que a incidência de litíase vesicular aumenta com o número de gestações ${ }^{23}$. Existe uma incidência aumentada em multíparas, quando comparadas com nulíparas.

Stauffer et al ${ }^{23}$ utilizando a ultra-sonografia durante a gravidez, evidenciaram um aumento no volume da vesícula biliar e uma redução na taxa de esvaziamento após a ingestão

\section{Tabela 8}

No grupo I: formado por todas as pacientes, pode-se constatar que:

Das características clínica a:

Idade: Em média, a cada ano aumenta em $4 \%$ a chance de se ter cálculo.

Parede da vesícula biliar espessada: $O$ fato de se ter parede espessada aumenta em 15 vezes a chance de se ter cálculo.

Paridade: Em média, cada passagem de nível $(0=0$ filhos; $1=1$ e 2 filhos; $2=3$ e 4 filhos; $3=$ mais de 4 filhos $)$ dobra a chance de se ter cálculo.

Dos achados ecográficos o:

Barro: O fato de se ter barro biliar aumenta em 11 vezes a chance de ser ter cálculo. 


\section{Tabela 9}

No grupo II: pacientes com menos de 50 anos pode-se constatar que:

Das características clínica a:

Paridade: Em média, a cada passagem de nível $(0=0$ filhos; $1=1$ e 2 filhos; $2=3$ e 4 filhos; $3=$ mais de 4 filhos $)$ dobra a chance de se ter cálculo.

Dos achados ecográficos o:

Parede da vesícula biliar espessada: $O$ fato de se ter parede espessada aumenta em 13 vezes a chance de ter litíase da vesícula biliar.

Barro: O diagnóstico ecográfico de barro biliar aumenta em oito vezes a chance de ser ter cálculo.

\section{Tabela 10}

No grupo III: pacientes com idade igual ou superior a 50 anos, pode-se constatar que:

Das características clínica a:

Idade: Em média, a cada ano, aumenta em $14 \%$ a chance de se ter cálculo.

Dos achados ecográficos o:

Barro: O diagnóstico ecográfico de barro biliar, aumenta em 26.5 vezes a chance de se ter cálculo.

de uma refeição. Sugerem esses autores que, devido a maior ocorrência de alteração no sistema biliar, o exame obstétrico deveria ser estendido sistematicamente a avaliação da vesícula biliar.

Analisando-se a prevalência de diabetes melitus nesta amostra, encontramos $9,1 \%$, sendo que, em $54,2 \%$, foi diagnosticada litíase vesicular. Esse percentual de cálculo em quem possui diabetes é significativamente superior do que no grupo não diabético, onde a incidência foi de $10.8 \%$.

A literatura refere maior incidência de litíase da vesícula biliar em pacientes diabéticos ${ }^{24}$. Esse fato pode ser relacionado com os hábitos dietéticos dos diabéticos Entretanto, a neuropatia autonômica, quando instalada, poderia ser responsável pela tendência litiásica nesse grupo de pacientes $^{24}$.

O deficiente esvaziamento vesicular pode ser fator importante. Esse fato pode estar relacionado a maior prevalência de litíase em gestante, idosos e diabéticos.

Devido à alta prevalência de litíase nos pacientes diabéticos, os nossos resultados conduzem a uma postura de que, havendo uma indicação para realização de exame ultrasonográfico, por um motivo qualquer, nesse grupo de pacientes, o exame deveria incluir a análise da vesícula biliar.

Para adequada compreensão da influência de todas as variáveis analisadas e sua relação com a litíase da vesícula biliar, ao se realizar uma análise de regressão logística multivariada, pode-se perceber o efeito que as variáveis exercem umas sobre as outras.

A variável diabetes não aparece no modelo final, em nenhum dos grupos. Tal análise causa questionamentos. Mas, analisando-se a relação diabetes e idade, constatou-se que a presença de diabetes e o seu percentual encontram-se fortemente concentrados nas idades superiores. Mais de $60 \%$ acima de 50 anos, portanto, essas variáveis possuem uma alta correlação, e uma capta o efeito da outra na ocorrência de litíase da vesícula biliar.

A litíase vesicular assintomática é uma doença com levada incidência, sujeita a inúmeras complicações, as quais, por sua vez, encerram elevado potencial de morbidade e mortalidade.

Certamente, diante do exposto, justifica-se plenamente um interesse maior em se estudar litíase vesicular assintomática em mulheres. $\mathrm{O}$ melhor entendimento de sua epidemiologia poderá modificar os atuais conhecimentos e permitir estabelecer-se uma abordagem cirúrgica em condições mais adequadas, do que quando ocorre sintomatologia e/ou complicações.

A relevância clínica do presente estudo reside, principalmente, no fato da maior prevalência de litíase vesicular assintomática em mulheres grandes multíparas, acima de 50 anos e diabéticas (fatores avaliados individualmente ou em associação). Uma vez que essas mulheres realizem exames ecográficos por qualquer outra indicação, seria prudente a avaliação da vesícula biliar quanto a pesquisa de marcadores ecográficos, facilmente identificáveis, que se associam mais frequentemente a litíase biliar, como é o caso da maior espessura da parede vesicular anterior, presença de barro biliar e aumento de sua medida transversal. Dessa forma, seria possível, como mencionado anteriormente, aprimorar o conhecimento na melhor forma de condução desses casos assintomáticos.

O entendimento da etiopatogenia e prevalência das doenças que, freqüentemente, atingem as mulheres, em qualquer faixa etária, devem sempre despertar a curiosidade, o interesse e a realização de pesquisas por parte dos cirurgiões e daqueles que exercem a prática ultra-sonográfica.

A presença de litíase vesicular assintomática nesta amostra esteve associada a: diabetes, paridade, idade, presença de barro biliar e a presença de espessamento da parede 
da vesícula biliar. A presença de litíase vesicular assintomática, em um serviço de ultra-sonografia de Ribeirão Preto, São Pau- lo, Brasil, nas pacientes submetidas a exames ultrasonográficos abdominal, vaginal e obstétrico, é de 14,7\%.

\begin{abstract}
Background: Our objective is to analyze ultrasonographic and clinical variables such as the presence or absence of bile sludge, thickness of the gallbladder wall, transverse measurement of the gallbladder, age, parity, and presence or absence of diabetes mellitus and to determine the prevalence of asymptomatic gallstones in female patients submitted to ultrasonographic examination. Methods: A prospective study was conducted on 265 female patients attended at the Ultrasonographic and Medical Updating School of Ribeirão Preto during the period from January to September 2001. Results: There was a statistically significant difference in gallstones and gallbladder wall thickness, bile sludge, transverse gallbladder diameter, age range, parity, from $4.1 \%$ among nulliparous women to $39.1 \%$ in multiparous women and women with diabetes. The prevalence of gallstones in asymptomatic patients was $14.7 \%$. Conclusion: The asymptomatic gallstones in women were mainly related to age and parity. The presence of galbladder wall thickness and bile sludge were the most frequent ultrasographic findings
\end{abstract}

Key words: Lithiasis; Gallbladder; Gallstones; Ultrasonography.

\section{REFERÊNCIAS}

1. Rhomberg HP, Judmair G, Lochs A. How common are gallstones? Br Med J (Clin Res Ed). 1984;289(6450):1002.

2. Chapman BA, Frampton CM, Wilson IR, Chisholm RJ, Allan $\mathrm{RB}$, Burt MJ. Gallstone prevalence in Christchurch: risk factors and clinical significance. N Z Med J. 2000;113(1104):46-8.

3. França LA, Santos ET, Carvalho AM, Silva AM, Rocha A. Prevalência de litíase biliar em cirróticos: avaliação necroscópica. Arq Gastroenterol. 1994; 31(3):92-6.

4. Ferreira AC. Mauad-Filho F, Carrara H, Elias Jr J, Barbosa Jr AB. Contribuição da ultra-sonografia abdominal no seguimento de pacientes pós-tratamento do câncer mamário. Radiol Bras. 2002;35(6):345-9.

5. Badalamenti S, DeFazio C, Castelnovo C, Sangiovanni A, Como G, De Vecchi A, Graziani G, Colombo M, Ponticelli C. High prevalence of silent gallstone disease in dialysis patients. Nephron. 1994;66(2):225-7.

6. Gibney EJ. Asymptomatic gallstones. Br J Surg. 1990;77(4):36872.

7. Heaton KW, Braddon FE, Mountford RA, Hughes AO, Emmett PM. Symptomatic and silent gall stones in the community. Gut. 1991;32(3):316-20.

8. Akute OO, Marinho AO, Kalejaiye AO, Sogo K. Prevalence of gall stones in a group of antenatal women in Ibadan, Nigeria. Afr J Med Med Sci. 1999;28(3-4):159-61.

9. Coelho JCU, Freitas AT, Fontan RS, Campos AC, Zeni Neto C, Oliva LV. Incidência de colesterolose da vesícula biliar em autópsias. Rev Col Bras Cir. 1993;20(6):295-7.

10. Coelho JCU, Frare RC, Arce VFL, Pitaki SA, Vitola ML, De George MA. Prevalência de litíase vesicular em pacientes hospitalizados em Curitiba: avaliação ultra-sonográfica. AMB Rev Assoc Med Bras. 1991;37(4):169-72.

11. Coelho JC, Bonilha R, Pitaki AS, Cordeiro RM, Salvalaggio PR, Bonin EA, Hahn CG, Soares RV, Milcheski DA. Prevalence of gallstones in a Brazilian population. Int Surg. 1999;84(1):25-8.

12. Nahrwold, DL. O sistema biliar. In: Sabiston DC. Tratado de cirurgia. $15^{\text {a }}$ ed. Rio de Janeiro: Guanabara Koogan; 1999. p. 1039-71.

13. Franco D, Roudie J. [Gallstones and their complications]. Rev Prat 2000;50(19):2117-22.
14. Achord JL. Are all gallstones «silent» until acute cholecystitis occurs? Gastroenterology. 1989;97(6):1591-2.

15. Jukemura J, Leite KRM, Machado MCC, Montagnini AL, Penteados S, Abdo EE, Cunha JE, Pinotti HW. Frequency of incidental gallbladder carcinoma in Brazil. ABCD Arq Bras Cir Dig. 1997;12(1/2):10-3.

16. Tazuma S, Kajiyama G. Carcinogenesis of malignant lesions of the gall bladder. The impact of chronic inflammation and gallstones. Langenbecks Arch Surg. 2001;386(3):224-9.

17. Wacha H, Ungeheuer E. [Symptomless gallstone disease-when to treat surgically?] Zentralbl Chir. 1987;112(13):843-8.

18. Trivino T, Lobo EJ, Goldenberg A. Doença biliar calculosa. In: Miszputen SJ. Guias de medicina ambulatorial e hospitalar UNIFESP/ Escola Paulista de Medicina-Gastroenterologia. 1a ed. São Paulo: Manole; 2002. p 337-44.

19. Patino JF, Quintero GA. Asymptomatic cholelithiasis revisited. World J Surg. 1998;22(11):1119-24.

20. Bapaye MY. Identifying asymptomatic patients with gallstones for treatment. Indian J Gastroenterol. 1997;16(2):75.

21. Filho IJ. Tratamento cirúrgico da colecistite crônica. In: Petroianu A. Terapêutica cirúrgica 1 ${ }^{\mathrm{a}}$. ed. Rio de Janeiro: Guanabara Koogan; 2001. p 321-347.

22. Everhart JE. Gallstones and ethnicity in the Americas. J Assoc Acad Minor Phys. 2001;12(3):137-43.

23. Stauffer RA, Adams A, Wygal J, Lavery JP. Gallbladder disease in pregnancy. Am J Obstet Gynecol. 1982;144(6):661-4.

24. Kumar D. Diabetic autonomic neuropathy causing gall bladder dysfunction. J Assoc Physicians India. 2001; 49:1042.

Como citar este artigo:

Ferreira AC, Mauad Filho F, Mauad FM, Barra DA, Mattos RL, Jorge Filho I. Litíase vesicular assintomática em mulheres: aspectos epidemiológicos e clínicos. Rev Col Bras Cir. [periódico na Internet] 2006 Jul-Ago;33(4). Disponível em URL: www.scielo.br/rcbc

Endereço para correspondência:

Adilson Cunha Ferreira

Rua Manoel Ache 9802- apto. 222.

Jardim Irajá

14020-590 - Ribeirão Preto - SP.

acferei@keynet.com.br 